\title{
Does the truth interfere with our ability to deceive?
}

\author{
Magda Osman \\ University of Surrey, Guildford, England \\ and University College London, London, England \\ AND \\ Shelley Channon and Sian FitzPatrick \\ University College London, London, England
}

\begin{abstract}
Does the truth interfere with our ability to respond deceptively? We considered this question in the present study by examining the effects of a task set (i.e., selecting truthful or untruthful responses), both by comparing two presentations of the same task and through transfer to a different task. All participants carried out the task either under the instructions to respond both correctly and incorrectly (Experiment 1) or under the instructions to respond truthfully and deceptively (Experiment 2); the order of instructions was counterbalanced. In Experiment 2, after completing the main task, the participants also performed a Stroop task. The findings suggested that deceptive responses took longer overall, regardless of the order of instructions. Moreover, the experience of responding deceptively versus truthfully led to faster responding on a subsequent Stroop test. Although there may well be processes unique to deceptive responding, the evidence suggests that overcoming interference is a process shared by deceptive and nondeceptive tasks.
\end{abstract}

The study of deceptive communication has revealed that when making a deceptive response (e.g., saying "No" to the question "Do you smoke?" when in fact you do), people take longer than when responding truthfully (see, e.g., Vendemia, Buzan, \& Green, 2005; Walczyck, Roper, Seemann, \& Humphrey, 2003; Walczyck et al., 2005). Slower processing is well documented in deception (see, e.g., DePaulo et al., 2003). For instance, in generating a lie, people typically remain close to the truth and omit or distort critical details (e.g., DePaulo et al., 2003). It seems that few people take the risk of generating a new account from scratch, and if they do, this is often aided by recruiting from memory other relevant experiences that can replace the truth (e.g., Johnson \& Raye, 1981) and that are often highly associated with it (e.g., Sporer, 2004). The truth is thought to provide a marker for the credibility of the constructed lie, helping the deceiver to attenuate his or her guilt by rationalizing that the deceptive communication is "virtually true" (e.g., Bond \& DePaulo, 2006).

Some (e.g., Jokinen, Santtila, Ravaja, \& Puttonen, 2006; Vendemia et al., 2005; Walczyck et al., 2003; Walczyck et al., 2005) have claimed that for deceptive responding, the presence of the truth is disadvantageous. Walczyck et al. (2005) proposed that relevant truthful memories that are triggered when to-be-lied-to questions are presented may interfere with deceptive responding. They found that distance in time (remote or recent memories) influ- enced the degree of memory activation. The accessibility of true memories may therefore have a direct interfering influence on the speed of deceptive responding. Extra controlled processes are thus needed to prevent the truth from interfering, whether through the downgrading or the suppression of the truthful response, and/or increased activation of the deceptive response through a selective attention mechanism (see, e.g., Egner \& Hirsch, 2005; Gorfein \& MacLeod, 2007; Spence et al., 2004; Vendemia et al., 2005). In support, recent neuroimaging evidence has revealed that activations when deceptive responses are made are similar to those reported in studies examining high loading of executive functions (e.g., Langleben et al., 2005; Nunez, Casey, Egner, Hare, \& Hirsch, 2005).

Thus far, it still remains unclear what task characteristics influence any interfering effects of the truth in responding deceptively. To investigate this question, we considered it from two perspectives: the degree of distance of the deceptive response from the truth, and the task set (namely, selecting truthful or untruthful responses). The latter was examined at two levels: within blocks of the same task, and through transfer to a different task.

We considered first whether selecting response options that are closely semantically related to the truth interferes more than does selecting those that are distantly related to the truth, on the basis of Walczyck et al.'s (2005) model. Using procedures similar to those used in forced choice

M. Osman,m.osman@ucl.ac.uk 
deception studies (Vendemia et al., 2005; Walczyck et al., 2003; Walczyck et al., 2005), we examined the generality of the interference effects by exploring them under both neutral (correct/incorrect; Experiment 1) and deceptionbased (true/lie; Experiment 2) instructions.

Second, we investigated whether the experience of developing a task set to respond truthfully hinders or facilitates responding deceptively, under both neutral and deceptive instructions. The development of a task set involves establishing a pattern of responding (Mayr \& Kliegl, 2000), and switching to an alternative task set involves overcoming interference from any previous set (see, e.g., Rogers \& Monsell, 1995). Third, we investigated another possible way that a task set may develop, by asking whether overcoming interference from an unwanted response is a general process found in both deceptive and nondeceptive tasks, sharing similar processing requirements to tasks such as the Stroop (1935) test. This test typically involves presenting color-word stimuli in conflicting ink colors (e.g., "blue" in red ink) (incongruent trials), or in consistent ink colors (e.g., "blue" in blue ink) (congruent trials). Typically, participants are slower to identify either the word or the ink color when there is a mismatch between them. If making deceptive responses represents a form of incongruency, similar processes may be involved in overcoming interference both in deception-based forced choice responding and in a Stroop task. We might then expect task-specific training gains, so that greater experience of deceptive responding should facilitate faster Stroop performance.

\section{EXPERIMENT 1}

In Experiment 1, we examined the first hypothesisthat the presence of response alternatives that are semantically proximal to versus distant from the correct (truthful) response may produce greater interference effects, and if so, that these effects may be greater when incorrect responses are made, versus correct responses. We also examined the second hypothesis relating to the within-task set by seeing whether the experience of correct responding interferes with subsequent incorrect responding.

\section{Method}

Participants. Seventy-nine (62 females) University College London (UCL) first-year undergraduate students (mean age = 19.98 years) took part in the experiment.

Design and Procedure. The task consisted of 10 different storybased scenarios, and there were eight questions relating to each one. For each scenario, participants read a short description of an event that had occurred, and this remained on-screen until all eight corresponding questions were presented and answered. Participants indicated that they were ready to proceed after reading the scenario, which prompted the presentation of a question. When they indicated that they were ready, two response alternatives appeared on the bottom left- and right-hand of the screen, and timing began. Once a choice was made, timing stopped, and the question and response alternatives were removed from screen. This procedure followed for all eight questions for each scenario.

Example of an item: Katie waited nervously behind the stage before her audition. She had been practicing her lines the night before, but was worried that she might forget them. She waited for them to call her name before putting her script back in her handbag and entering the room.
Example of a question containing a correct versus distally related incorrect response option:

Katie waited nervously behind the? Box office or Stage

Example of a question containing a correct versus proximally related incorrect response option:

Katie waited nervously behind the? Curtain or Stage

For each scenario, four pairs of questions were developed, each comprising a correct versus distally related incorrect response option, and a correct versus proximally related incorrect response option. Doing this produced eight questions in all, for each of the 10 scenarios. The position of the response alternatives (left or right) was randomized across questions. The order of presentation of the eight questions was pseudorandomized for each scenario so that no pair of related questions appeared consecutively.

All of the participants performed the 10-scenario task twice, once under instructions to give correct answers, and once under instructions to give incorrect answers. Participants were randomly assigned to one of two instruction conditions: either correct-incorrect (respond correctly throughout the first task presentation, then incorrectly throughout the second task presentation) $(n=41)$, or incorrect-correct (respond incorrectly first, then correctly second) $(n=38)$. Thus, when responding correctly, participants were required to respond selectively to the correct option and to ignore the incorrect option (either proximal or distal), and when responding incorrectly, they were required to respond only to the incorrect option (either proximal or distal) and to ignore the correct option.

\section{Results and Discussion}

Mean reaction times (RTs, in milliseconds) for distal and proximal response selections by instructions and order are presented in Figure 1. Errors in response selections were infrequent, and statistical analyses revealed no significant difference between failing to select distal $(0.63 \%)$ and proximal $(1.64 \%)$ response alternatives, and selecting the distal $(2.15 \%)$ and proximal $(3.79 \%)$ distractors. All analyses that follow are based on responses without errors, using logarithmically transformed data.

Semantic distance. Consistent with previous evidence (Vendemia et al., 2005; Walczyck et al., 2003; Walczyck et al., 2005), there was a significant main effect of truth $\left[F(1,77)=44.97, p<.0005, \eta_{\mathrm{p}}^{2}=.37\right]$; responding correctly was faster than responding incorrectly. In order to ex-

\section{Mean RTs by Instructions and Response Distance in Experiment 1}

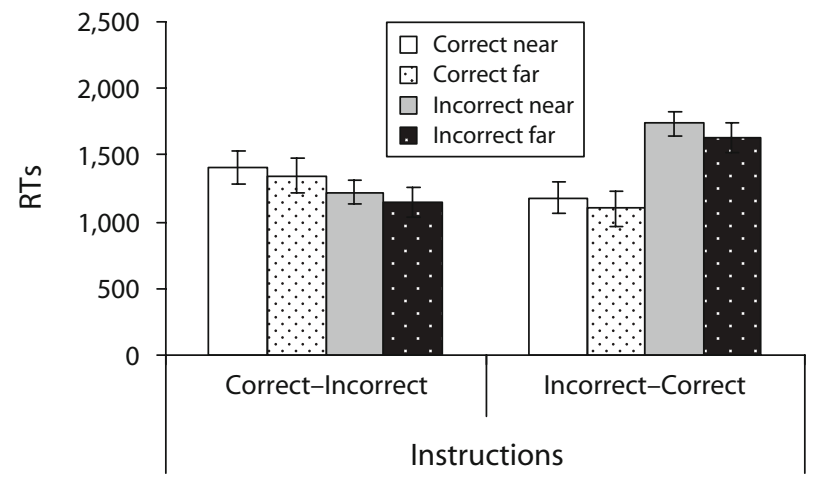

Figure 1. Mean response times (RTs, in milliseconds) and standard errors of the mean by instructions and response distance in Experiment 1. 
amine any effect of semantic distance from the correct answer (truth) on incorrect responding, a $2 \times 2 \times 2$ ANOVA was conducted with semantic distance (distal, proximal) and truth (correct, incorrect) as within-group factors, and instructions (correct-incorrect, incorrect-correct) as a between-groups factor. There was a main effect of semantic distance $\left[F(1,77)=39.25, p<.0005, \eta_{\mathrm{p}}^{2}=.34\right]$, showing that the presence of semantically proximal rather than distal information slowed responses. However, since there were no significant interactions involving semantic distance, participants were slowed regardless of whether they were ignoring the proximal response alternative (when instructed to respond correctly) or selecting the proximal response alternative (when instructed to respond incorrectly).

Within-task task set. The effects of performing the task twice were examined with respect to the effects of prior experience of correct (truthful) responding on performance. There was a significant main effect of instructions $\left[F(1,77)=4.33, p<.041, \eta_{\mathrm{p}}^{2}=.05\right]$, showing that those in the correct-incorrect condition responded faster overall than did those in the incorrect-correct condition. There was also a significant truth $\times$ instructions interaction $\left[F(1,77)=224.88, p<.0005, \eta_{\mathrm{p}}^{2}=.75\right]$.

It can be seen from Figure 1 that both groups speeded up from the first to the second completion of the task, and that this effect was larger for the group switching from incorrect to correct responding. A $t$ test comparison of incorrect responses (averaged across semantic distance) for the correct-incorrect group versus the incorrect-correct group showed that these were slower for the latter participants $[t(77)=7.84, p<.0005$, Cohen's $d=1.85]$, suggesting that acquiring a task set for responding truthfully facilitated rather than interfered with subsequent incorrect responding, presumably reflecting some general effect of practice on the task.

\section{EXPERIMENT 2}

Experiment 2 was designed to replicate and extend Experiment 1 by using deception-based instructions instead of neutral instructions, so that participants were told to select the "lie" rather than the "incorrect" response, and to select the "true" rather than the "correct" response. In addition, the results of Experiment 1 suggested that prior practice at the task was sufficient to facilitate performance. To examine the second hypothesis further by distinguishing between the effects of practice versus a specific task set, in Experiment 2, we included two additional conditions in which participants were instructed to lie or to respond truthfully for both the first and second presentations of the task. Finally, in Experiment 2, we also explored the third hypothesis: the potential relationship between responding deceptively and any effects of transfer in overcoming later interference. To explore this, we included a version of the Stroop task at the end of the deception-based task. It was hypothesized that if there are shared processes involved in the two tasks, then experience of deceptive responding should facilitate performance on the Stroop task, particularly on incongruent trials.

\section{Method}

Participants. Sixty-four (50 females) UCL first-year undergraduate students (mean age $=20$ years, $S D=2.65$ ) took part in the experiment.

Design and Procedure. With the following three exceptions, in all other respects, Experiments 1 and 2 were identical. First, the instructions directed participants to respond truthfully or to lie rather than to respond with the correct or incorrect answer (true-lie or lie-true). Second, two additional practice conditions were added in which instructions for the first and second task completions were the same, involving either only truthful (true-true) or deceptive (lie-lie) responses. Participants were randomly assigned to one of these four conditions: true-true, lie-true, true-lie, and lie-lie, with 16 participants in each condition.

Third, after completing the task twice, all participants carried out a Stroop task. A total of 40 congruent (i.e., ink and word compatible) and 40 incongruent (i.e., ink and word incompatible) trials were presented. Half of the trials instructed participants to name the color of the ink, regardless of the word itself, and the remainder instructed them to name the word, regardless of the ink color. The order of the presentation of word versus ink trials was counterbalanced across participants.

\section{Results and Discussion}

As for Experiment 1, the frequency of errors for the main deception task was low, and analyses revealed no significant difference between responding incorrectly by failing to select distal $(2.04 \%)$ and proximal $(2.34 \%)$ response alternatives, and by incorrectly selecting the distal $(1.87 \%)$ and proximal $(2.96 \%)$ distractors. The following analyses are based on correct RT responses, using logarithmically transformed data.

Semantic distance. A $2 \times 2 \times 2 \times 2$ ANOVA was carried out using the data from all four conditions in Experiment 2, with semantic distance (distal, proximal) and repetition (first or second task completion) as within-group factors, and instructions (true or lie on first task completion) and second instructions (true or lie on second task completion) as between-groups factors (see Table 1A). There was a significant main effect of semantic distance $\left[F(1,60)=46.67, p<.0005, \eta_{\mathrm{p}}^{2}=.44\right]$, consistent with the results of Experiment 1, showing that the presence of semantically proximal rather than distal information slowed responses. However, as in Experiment 1, there were no significant interactions involving semantic distance (see Figure 2).

Within-task task set. The ANOVA also showed a significant main effect of repetition $[F(1,60)=124.53$, $\left.p<.0005, \eta_{\mathrm{p}}^{2}=.68\right]$, suggesting that overall, decisions were faster on the second completion of the task than on the first. The results of Experiment 1 suggested that incorrect responding was facilitated by task practice, since those who had previously completed the task under correct instructions were faster than those with no prior task practice. However, Experiment 1 could not differentiate between general task practice and any specific task set. To address this, we compared deceptive performance for those who had previously completed the task under instructions to tell the truth (general task practice and opposite task set) with that for those who had previously completed it under instructions to lie (general task practice and same task set) (see Figure 2). Although the lie-lie 
Table 1A

Mean Scores and Standard Deviations for the Story Task in Experiments 1 and 2

\begin{tabular}{|c|c|c|c|c|c|c|c|c|}
\hline \multirow[b]{3}{*}{ Instructions } & \multicolumn{4}{|c|}{ Correct/Truthful Alternative } & \multicolumn{4}{|c|}{ Incorrect/Deceptive Alternative } \\
\hline & \multicolumn{2}{|c|}{$\begin{array}{c}\text { Proximal Semantic } \\
\text { Distance }\end{array}$} & \multicolumn{2}{|c|}{$\begin{array}{l}\text { Distal Semantic } \\
\text { Distance }\end{array}$} & \multicolumn{2}{|c|}{$\begin{array}{l}\text { Proximal Semantic } \\
\text { Distance }\end{array}$} & \multicolumn{2}{|c|}{$\begin{array}{l}\text { Distal Semantic } \\
\text { Distance }\end{array}$} \\
\hline & $M$ & $S D$ & $M$ & $S D$ & $M$ & $S D$ & $M$ & $S D$ \\
\hline \multicolumn{9}{|c|}{ Experiment 1} \\
\hline Correct-Incorrect & $1,410.56$ & 351.26 & $1,344.11$ & 317.61 & $1,218.85$ & 275.93 & $1,146.40$ & 236.72 \\
\hline Incorrect-Correct & $1,180.67$ & 250.88 & $1,098.33$ & 211.28 & $1,737.94$ & 367.62 & $1,627.48$ & 358.49 \\
\hline \multicolumn{9}{|c|}{ Experiment 2} \\
\hline True-Lie & $1,442.57$ & 422.15 & $1,311.07$ & 362.67 & $1,341.44$ & 344.95 & $1,259.56$ & 332.34 \\
\hline Lie-True & $1,354.22$ & 515.19 & $1,217.9$ & 400. & $2,030.19$ & 536.47 & $1,707.90$ & 396.16 \\
\hline True-True & $1,621.03$ & 428.29 & $1,372.07$ & 291. & $1,072.68$ & 195.95 & $1,011.99$ & 138.95 \\
\hline Lie-Lie & $1,764.35$ & 513.38 & $1,650.28$ & 528.06 & $1,414.94$ & 387.36 & $1,288.23$ & 306.30 \\
\hline
\end{tabular}

Table 1B

Mean Scores and Standard Deviations for the Stroop Task in Experiment 2 Only

\begin{tabular}{|c|c|c|c|c|c|c|c|c|}
\hline \multirow[b]{3}{*}{ Instructions } & \multicolumn{4}{|c|}{ Congruent } & \multicolumn{4}{|c|}{ Incongruent } \\
\hline & \multicolumn{2}{|c|}{ Ink } & \multicolumn{2}{|c|}{ Word } & \multicolumn{2}{|c|}{ Ink } & \multicolumn{2}{|c|}{ Word } \\
\hline & $M$ & $S D$ & $M$ & $S D$ & $M$ & $S D$ & $M$ & $S D$ \\
\hline True-Lie & $1,105.58$ & 573.46 & 804.00 & 390.05 & $1,205.68$ & 465.49 & $1,093.64$ & 625.79 \\
\hline Lie-True & $1,094.48$ & 411.30 & 898.79 & 317.64 & $1,158.89$ & 341.91 & $1,159.92$ & 435.14 \\
\hline True-True & $1,218.47$ & 415.19 & 985.71 & 405.51 & $1,318.50$ & 387.35 & $1,141.60$ & 438.81 \\
\hline Lie-Lie & $1,031.13$ & 300.52 & 793.44 & 207.83 & $1,040.75$ & 281.23 & 942.17 & 184.40 \\
\hline
\end{tabular}

group was significantly slower than the true-lie group on the first completion of the task $[t(30)=2.16, p<.039$, Cohen's $d=.76$ ] (i.e., lying took longer than telling the truth), there was no significant difference on the second task completion in the speed of deceptive responding between these two groups, suggesting that performance was affected primarily by general task practice rather than by a specific task set.

Effects of a task set involving deceptive experience on the Stroop task. To what extent does the experience of deceptive responding influence responding on a subsequent interference task? The Stroop task was performed after the story task to examine the influence of prior practice in

\section{Mean RTs by Instructions and Response Distance in Experiment 2}

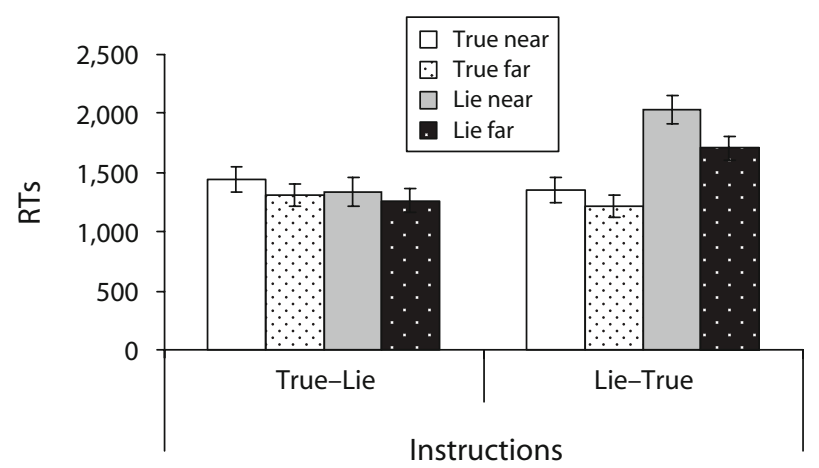

Figure 2. Mean response times (RTs, in milliseconds) and standard errors of the mean by instructions and response distance in Experiment 2. overcoming interference. Mean RTs for this are presented in Table 1B. A $2 \times 2 \times 2 \times 2$ ANOVA with congruency (congruent, incongruent trials) and inkword (respond-toink-color or respond-to-word-meaning) as within-group factors, and instructions (true or lie on first completion of story task) and second instructions (true or lie on second completion of story task) as between-groups factors.

The Stroop data showed the expected significant effect of congruency $\left[F(1,60)=66.36, p<.0005, \eta_{\mathrm{p}}^{2}=\right.$ .53], since responses to congruent trials were faster than were those to incongruent trials, and the expected significant effect of inkword $[F(1,60)=40.44, p<.0005$, $\left.\eta_{\mathrm{p}}^{2}=.40\right]$, since responding to the dominant word meaning was faster than responding to the ink color. There was also a significant inkword $\times$ congruency interaction $\left[F(1,60)=16.06, p<.0005, \eta_{\mathrm{p}}^{2}=.21\right]$, and no other interactions involving inkword; a post hoc paired $t$ test showed that the difference between incongruent and congruent trials was greater for respond-to-word than for respond-to-ink-color instructions $[t(63)=4.03, p<$ .0005 , Cohen's $d=.72]$.

With respect to the effects of prior deceptive experience on Stroop responding, there were no significant main effects of instructions or second instructions, nor was there an interaction between them, and there were no interactions with inkword; there was a significant congruency $X$ instructions $\times$ second instructions interaction $[F(1,60)=$ $\left.4.71, p=.034, \eta_{\mathrm{p}}^{2}=.07\right]$. Mean scores showed that, as was predicted, the experience of responding deceptively facilitated Stroop performance (see Figure 3). Direct post hoc comparison of the two practice groups combining across ink color and word trials showed that incongruent (but not congruent) responses on the Stroop task were signifi- 


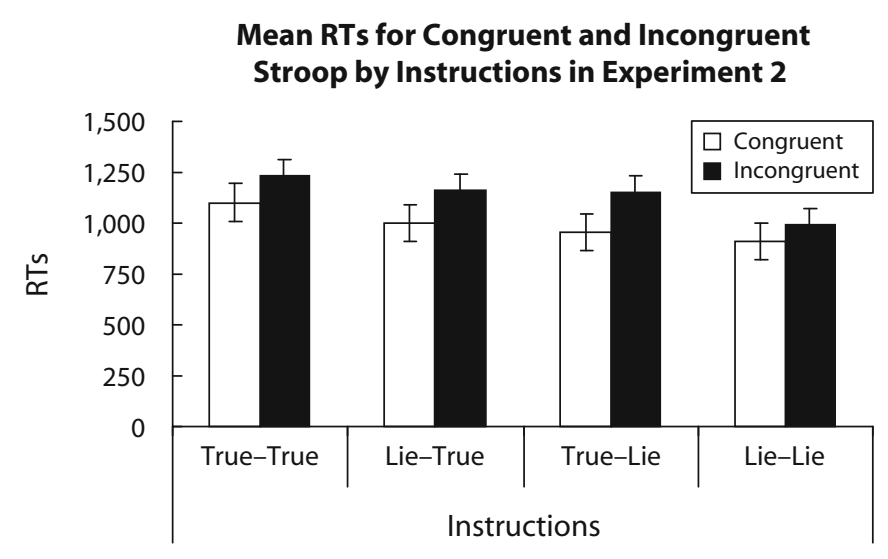

Figure 3. Mean response times (RTs, in milliseconds) and standard errors of the mean for congruent and incongruent Stroop trials averaged across ink and word stimuli, by task instruction in Experiment 2.

cantly faster for those with the greatest prior experience of deceptive responding (lie-lie group) versus those with no prior experience of deceptive responding (true-true group) $[t(30)=2.15, p<.040$, Cohen's $d=.76]$.

\section{GENERAL DISCUSSION}

The present study was designed to examine the way in which the truth interferes with responding deceptively. On the basis of Walczyck et al.'s (2005) proposal that the activation of recent truthful memories interferes more than do remote memories with the speed of deceptive responding, we considered a related issue - namely, whether selecting response options that are closely semantically related to the truth interferes more than does selecting those that are distantly related to the truth. Our findings did not support the notion of a specific interfering effect of truthful information on deceptive responding. Instead, the results showed semantic distance effects that were independent of task instruction, since both truthful and incorrect/deceptive responses were slower when the incorrect response alternative was closer to the truth. However, one consideration is that in Walczyck et al.'s (2003) and Walczyck et al.'s (2005) forced choice studies, the items were self-generated and personal to the individual, whereas in the present study, they were not. It may be the case that the relationship between semantic distance effects and deceptive responding is enhanced in highly personalized situations. One way in which this could be addressed is to use a task design similar to the one developed in the present study, but to include scenarios that are personal to the individual, and in which they generate the proximal and distal options.

Is it crucial that participants are intending to deceive, or is the critical factor that they are instructed to choose an incorrect answer? A similar pattern of responding was found across two experiments under both neutral (correctincorrect) and deception-based (true-lie) instructions. It is of course possible that participants do not perceive themselves to be actively engaged in deception unless they generate their own responses, rather than select from predetermined ones, as in the present study. However, face-to-face lying is also associated with slowed responding (for discussion, see DePaulo et al., 2003), and a number of previous studies in the deception literature have also used computer-based choice reaction tests in order to examine deceptive responding (see, e.g., Nunez et al., 2005; Spence et al., 2004; Vendemia et al., 2005; Walczyk et al., 2003; Walczyk et al., 2005). We contend that interference effects may play an important role in the selection of deceptive responses, and that these are unlikely to be specific to deception tasks.

Interference effects were also examined in the present study in relation to task set, both within blocks of the same task and through transfer to a different task. Within blocks of the same task, the findings suggest that developing a task set for responding truthfully (by performing the task first under instructions to select truthful responses) does not in itself interfere with responding deceptively; indeed, there may even be a facilitatory role. Faster performance on the second presentation of the task was found across instructional orders on the two experiments. The most parsimonious explanation for this is an effect of task practice.

With respect to transfer to a different task, both the deception and Stroop tasks involved overcoming the interfering effects of task-irrelevant items. We postulated that if processes involved in overcoming interference contributed to slower deceptive responding, experience of this type of response in the deception task should facilitate performance on an unrelated task that requires similar processing. The clearest support for this was provided by a comparison of the two conditions in Experiment 2 that gave either no experience (true-true) or a maximal experience (lie-lie) of deceptive responding. Practice in responding deceptively rather than truthfully was found to enhance performance on an unrelated Stroop task. This facilitation effect suggests that the development of a general task set to respond deceptively may require selective attention to items relevant to the task at hand, while overcoming interference from task-irrelevant items. Recent work by Dahlin, Neely, Larsson, Bäckman, \& Nyberg (2008) has suggested that the transfer of learning effects can be seen on working memory tasks, but only if they are 
mediated by the same neural system. A similar approach could be taken to elucidate more fully the general underlying processes involved in deceptive responding, by comparing any transfer effects for subsequent tasks that are thought to share the same underlying processes, as well as for other tasks that do not.

\section{AUTHOR NOTE}

The support of the ESRC Research Centre for Economic Learning and Social Evolution (ELSE) is gratefully acknowledged. Correspondence concerning this article should be addressed to M. Osman, Department of Psychology, University of Surrey, Guildford, Surrey GU2 7XH, England (e-mail: m.osman@ucl.ac.uk).

\section{REFERENCES}

Bond, C. F., \& DePaulo, B. (2006). Accuracy of deceptive judgments. Personality \& Social Psychology Review, 10, 214-234.

Dahlin, E., Neely, A. S., Larsson, A., Bäckman, L., \& Nyberg, L. (2008). Transfer of learning after updating training mediated by the striatum. Science, 320, 1510-1512.

DePaulo, B. M., Lindsay, J. J., Malone, B. E., Muhlenbruck, L., Charlton, K., \& Cooper, H. (2003). Cues to deception. Psychological Bulletin, 129, 74-118.

Egner, T., \& Hirsch, J. (2005). Cognitive control mechanisms resolve conflict through cortical amplification of task-relevant information. Nature Neuroscience, 8, 1784-1790.

Gorfein, D. S., \& MacLeod, C. M. (2007). Inhibition in cognition. Washington, DC: American Psychological Association.

Johnson, M. K., \& RaYe, C. L. (1981). Reality monitoring. Psychological Review, 88, 67-85.

Jokinen, A., Santtila, P., Ravaja, N., \& Puttonen, S. (2006). Salience of guilty knowledge test items affects accuracy in realistic mock crimes. International Journal of Psychophysiology, 62, 175-184.
Langleben, D. D., Loughead, J. W., Bilker, W. B., Ruparel, K. Childress, A. R., Busch, S. I., \& GuR, R. (2005). Telling truth from lie in individual subjects with fast event-related fMRI. Human Brain Mapping, 26, 262-272.

MaYr, U., \& Kliegl, R. (2000). Task-set switching and long-term memory retrieval. Journal of Experimental Psychology: Learning, Memory, \& Cognition, 26, 1124-1140.

Nunez, J. M., Casey, B. J., Egner, T., Hare, T., \& Hirsch, J. (2005). Intentional false responding shares neural substrates with response conflict and cognitive control. NeuroImage, 25, 267-277.

Rogers, R. D., \& Monsell, S. (1995). Costs of a predictable switch between simple cognitive tasks. Journal of Experimental Psychology: General, 124, 207-231.

Spence, S. A., Hunter, M. A., Farrow, F. D., Green, R. D., Leung, D. H., Hughes, C. J., \& Ganesan, V. (2004). A cognitive neurobiological account of deception: Evidence from functional neuroimaging. Philosophical Transactions of the Royal Society B, 359, 1755-1762.

SPORER, S. L. (2004). Reality monitoring and the detection of deception. In P.-A. Granhag \& L. A. Strömwall (Eds.), The detection of deception in forensic contexts (pp. 64-102). Cambridge: Cambridge University Press.

Stroop, J. R. (1935). Studies of interference in serial verbal reactions. Journal of Experimental Psychology, 18, 643-662.

Vendemia, J. M., Buzan, R. F., \& Green, E. P. (2005). Practice effects, workload, and reaction time in deception. American Journal of Psychology, 118, 413-429.

WalczyK, J. J., Roper, K. S., Seemann, E., \& Humphrey, A. M. (2003). Cognitive mechanisms underlying lying to questions: Response time as a cue to deception. Applied Cognitive Psychology, 17, 755-774.

WalczyK, J. J., Schwartz, J. P., Clifton, R., Adams, B., Wei, M., \& ZHA, P. (2005). Lying person-to-person about life events: A cognitive framework for lie detection. Personnel Psychology, 58, 141-170.

(Manuscript received September 8, 2008; revision accepted for publication June 16, 2009.) 